\title{
Oxidative stress and antioxidative parameters in patients with spinal cord injury: implications in the pathogenesis of disease
}

\author{
G Fatima $^{1}$, VP Sharma ${ }^{2}$, SK Das ${ }^{3}$ and AA Mahdi ${ }^{1}$
}

Study design: Oxygen-derived free radicals have been implicated in the pathogenesis of spinal cord injury (SCl) after trauma. Objective: In this review we will elucidate the importance of oxidative stress and antioxidants and its possible relationship with SCl. Methods: Literature analysis of oxidative stress, antioxidative parameters based on its implications in the pathogenesis along with devastating effect of oxidative stress parameters on $\mathrm{SCl}$ patients and its suggested proposed treatment by antioxidants have been performed.

Results: SCI remains a major health problem despite advances in neurotechnology. Previous studies have reported oxidative stress in $\mathrm{SCl}$ patients, but the results were inconsistent. Furthermore, increased free radical levels are reported in SCl. Moreover, we have also mentioned in this review that oxidative stress is supposed to be increased in patients with $\mathrm{SCl}$, which is related to the severity of $\mathrm{SCl}$ pain.

Conclusion: Oxidative stress was commonly seen in $\mathrm{SCl}$ patients, which may provide useful information to augment the understanding of pathophysiology of SCl patients. However, complete understanding of the biochemical events occurring at a cellular level that influence oxidative damage is required to guide future therapeutic advances. Furthermore, supplementation of antioxidants may also be considered in these patients.

Spinal Cord (2015) 53, 3-6; doi:10.1038/sc.2014.178; published online 4 November 2014

\section{INTRODUCTION}

Spinal cord injuries (SCI) cause myelopathy or damage to nerve roots or myelinated fiber tracts that carry signals to and from the brain. The prevalence of SCI is not known in India and many other countries. According to the known data, about 450000 people in the United States live with SCI (1 in 670), and there are about 11000 new SCI every year $(1$ in 30000$)$. The events occurring immediately after SCI include neuronal fiber damage, mass ischemic, neural cell necrosis and apoptosis, the destruction of microvasculature, inflammation, lipid peroxidation (LP), free radical production, demyelination and glial scar formation, leading to extensive secondary tissue injuries. ${ }^{1}$ Robust cell death in the injured region happens from seconds to weeks after SCI, which results in the formation of the cavities or cysts that blocks ascending and descending neurotransmission. ${ }^{2}$ In response to local inflammation after SCI microglia and central nervous system (CNS)resident macrophages are activated, which then exacerbate the inflammatory reaction in the injured center. ${ }^{3}$ Accordingly, it is thought that the inflammatory reactions could take place over weeks after SCI, which induce the recruitment of neutrophils, macrophages and T cells from hours to weeks after injury. ${ }^{4}$

Most of the body's energy is produced by the enzymatically controlled reaction of oxygen with hydrogen in oxidative phosphorylation occurring within the mitochondria during oxidative metabolism. During this enzymatic reduction of oxygen to produce energy, a small amount of free radicals are formed. ${ }^{5}$ Radicals can provide signals to the cell for growth, immune response and regulatory redox reactions, but excessive amounts may damage proteins, lipids and DNA via oxidative reactions. In the normal condition there is a balance between reactive oxygen species (ROS) and antioxidants within the cell, in the membranes and in the extracellular space. Endogenous free radical scavengers, namely antioxidants, are overwhelmed by excessive production of ROS. The ROS attack the polyunsaturated fatty acids in the membrane lipids, thereby leading to LP resulting in the loss of fluidity of the membranes, changes in membrane potentials and eventually rupture leading to the release of cell and organelle contents. ${ }^{6}$ Therefore, increased oxidative stress results from an imbalance between products of oxidation and antioxidant defenses. These toxic molecules (molecules of ROS) become highly reactive in their formation because of their altered number of unpaired valence electrons. There are several inflammatory clinical conditions associated with increased oxidative stress, data also suggest a relationship between oxidative stress and pain perception. However, free radicals have an important role in the pathogenesis of $\mathrm{SCI}^{7}$

There is little information about oxidative stress in SCI. Moreover, there are several disorders associated with oxidative stress that are manifested by LP, protein oxidation and other markers. Studies have shown evidence that oxidative stress may have a role in the

${ }^{1}$ Department of Biochemistry, King George's Medical University, Lucknow, India; ²Department of Physical Medicine \& Rehabilitation, King George's Medical University, Lucknow, India and ${ }^{3}$ Department of Rheumatology, King George's Medical University, Lucknow, India

Correspondence: Dr G Fatima, Department of Biochemistry, King George's Medical University, C-2946, Lucknow 226003, India,

E-mail: ghizalfatima8@gmail.com

Received 24 March 2014; revised 8 September 2014; accepted 28 September 2014; published online 4 November 2014 
pathophysiology of SCI. Attempts have not been made on establishing oxidative and antioxidative association in SCI patients, which is essential for understanding the development of SCI. Therefore, in this review, we will try to elucidate the complex network of oxidative and antioxidative imbalance in SCI patients.

\section{OXIDATIVE STRESS}

Oxidative and nitrosative stress could be defined as an imbalance between the presence of high levels of ROS and reactive nitrogen species, and the antioxidative defense mechanisms. These toxic molecules are formed via oxidation-reduction reactions and are highly reactive as they have an odd number of electrons. ROS generated under physiological conditions are essential for life, as they are involved in bactericidal activity of phagocytes, and in signal transduction pathways, regulating cell growth and reduction-oxidation (redox) status. ${ }^{8}$ ROS includes free radicals, such as hydroxyl and superoxide radicals, and nonradicals, including hydrogen peroxide and singlet oxygen. Oxidative stress and generation of free radicals, as primary or secondary event, have been related in a great number of diseases. ${ }^{9}$ Like ROS, reactive nitrogen species could also have a significant role in the pathogenesis of many diseases, and have drawn significant attention in recent years. Nitric oxide (NO), generated by the enzyme inducible NO synthase, is one of the most important and widely studied reactive nitrogen species. Therefore, oxidative stress, arising as a result of an imbalance between free radical production and antioxidant defenses, is associated with damage to a wide range of molecular species including lipids, proteins and nucleic acids. Oxidative damage to proteins and nucleic acids similarly gives rise to a variety of specific damage products as a result of modifications of amino acids or nucleotides. ${ }^{10}$ Such oxidative damage might also lead to cellular dysfunction, and it might contribute to the pathophysiology of a wide variety of diseases including SCI.

\section{OXIDATIVE STRESS AND SCI}

Oxidative stress means an alteration in the delicate balance between free radicals and the scavenging capacity of antioxidant enzymes in favor of free radicals in the body systems. Recent studies have shown that oxidative stress may have a role in the pathophysiology of SCI. Further, one of the most investigated molecular mechanisms involved in the secondary pathophysiology of acute SCI is free radical-induced, iron-catalyzed LP and protein oxidative/nitrative damage to spinal neurons, glia, and microvascular cells. The reactive nitrogen species peroxynitrite $(\mathrm{PN})$ and its highly reactive free radicals are key initiators of LP and protein nitration in the injured spinal cord. ${ }^{11}$ Oxidant, antioxidant parameters have been studied in rats model induced with SCI in which the oxidative stress markers such as malondialdehyde (MDA) and advanced oxidation protein products (AOPP) were measured along with antioxidants such as glutathione (GSH) peroxidase, superoxide dismutase (SOD), catalase (CAT) and reduced GSH where they reported, significant increase in MDA, AOPP, SOD and GSH peroxidase and decrease in the GSH and CAT activities, and in conclusion they found that oxidative stress evidenced by increased MDA, AOPP and decreased GSH and CAT support the generation of ROS in rat model induced with SCI. ${ }^{12}$

Free radical-induced LP NO level and the level of oxidized protein products is believed to have an important role in the pathogenesis of secondary injury. In addition, free radicals cause the most destructive effect on the CNS with LP. NO is a highly diffusible and labile, gaseous messenger molecule, involved in various biological functions such as modulation of nociception, immune function and neurotransmission. Moreover, NO has a physiological role in neuronal signal transmission and vessel dilation and is also involved in the secondary injury that occurs following SCI. Furthermore, the NO in patients with SCI correlates with neurologic severity and recovery rate postoperatively, or with conservative treatment. ${ }^{13}$ An excessive NO production has cytotoxic effects and induces neuronal apoptosis secondary to neural degeneration and neurodysfunction. ${ }^{14}$

Several disorders are associated with oxidative stress that are manifested by LP, protein oxidation and other markers. A study by Liu et al. ${ }^{15}$ reported that oxidative stress levels increase markedly during the first weeks of strict immobilization after the onset of an SCI. In parallel, also 2-3 months of strict bed rest in healthy subjects causes an increase in oxidative stress, whereas returning to initial $a$ priori physical activity normalizes (anti) oxidative status. ${ }^{16}$ ROSinduced oxidative damage is a well-documented secondary injury mechanism after CNS injury, PN, formed by the diffusion rate-limited combination of $\mathrm{NO}$ and superoxide $\left(\mathrm{O}_{2}^{-}\right)$free radicals, has been proposed to be a key contributor to posttraumatic oxidative damage. ${ }^{17}$ LP is the main cause of the further secondary damage that starts after mechanical destruction of tissues. LP products increase immediately after SCI, and the peak concentrations of ROS and nitrogen species occur within the first $24 \mathrm{~h} .{ }^{18}$ A study by Hall et al. ${ }^{19}$ has proposed that the most effective approach to interrupt posttraumatic oxidative brain damage after traumatic brain injury might involve the combined treatment with mechanistically complementary antioxidants thatsimultaneously scavenge LP-initiating free radicals, and inhibit LP propagation, and finally remove neurotoxic LP byproducts. NO is a highly diffusible and labile, gaseous messenger molecule, involved in various biological functions such as vasodilatation or vascular regulation, modulation of nociception, immune function, neurotransmission and excitation-contraction coupling. ${ }^{20} \mathrm{NO}$ also acts as a metabolic regulator during exercise. ${ }^{20}$ Recently, $\mathrm{NO}$ has been shown to modulate levels of ROS in a variety of cells. ${ }^{21}$

Mitochondrial dysfunction has a critical role in the posttraumatic cell death cascade in the injured spinal cord. It is clear that this is directly related to the intracellular accumulation of calcium $\left(\mathrm{Ca}^{++}\right)$ ions, which compromise mitochondrial function and increase ROS production. Following SCI, there is a loss of mitochondrial homeostasis together with increased mitochondrial ROS production and LPinduced disruption of synaptic homeostasis. ${ }^{22}$ Evidence has accumulated, which shows a particularly important ROS that is being formed by $\mathrm{Ca}^{++}$stressed mitochondria is $\mathrm{PN}$. NO has been shown to be present in mitochondria derived from a mitochondrial NO synthase isoform. The relationship of PN generation to mitochondrial dysfunction in the injured spinal cord has been recently documented by studies that have shown that the timing of post-SCI mitochondrial dysfunction (that is, respiratory and $\mathrm{Ca}^{++}$buffering impairment) is correlated with an increase in PN induced 3-nitrotyrosine (3-NT), 4-hydroxynonenal (4-HNE) and protein carbonyl content in mitochondrial proteins. ${ }^{23}$ The loss of mitochondrial function and the increase in oxidative damage markers, including 3-NT, is antagonized by early in vivo postSCI treatment with the PN radical scavenger tempol. ${ }^{24}$

Other studies have demonstrated that the exposure of normal CNS mitochondria to 4-HNE rapidly impairs their respiratory function and that spinal cord mitochondria are 10-fold more sensitive to this effect than brain mitochondria. ${ }^{25}$ But, from a physiological point of view, what relationship exists between oxidative stress and the symptoms of $\mathrm{SCI}$ ? It is known that ROS are involved in the etiology of one of the major symptoms of SCI, and that is pain. A more novel factor is oxidative stress and/or decreased antioxidative capacity in the vascular wall. ${ }^{26}$ Possibly, an imbalance in (anti) oxidative status may contribute to the increased prevalence of cardiovascular diseases in SCI. 
These results suggest that ROS production in mitochondria may be involved in oxidative stress, and CoQ10 deficiency and that mitochondrial dysfunction may be also involved in the pathophysiology of SCI. These results confirm that the oxidative stress background of SCI is probably due to a defect in the antioxidant system (SOD, CoQ10) and a high production of ROS. Finding the origin of oxidative stress could help us to offer new therapeutic strategies for SCI patients.

\section{ANTIOXIDANTS}

Antioxidants are substances that can buffer or neutralize all ROS species. The physiological role of antioxidants, as this definition suggests, is to prevent damage to cellular components arising as a consequence of chemical reactions involving free radicals. In recent years, a substantial body of evidence has been developed supporting a key role for free radicals in many fundamental cellular reactions and suggesting that oxidative stress might be important in the pathophysiology of common diseases including atherosclerosis, chronic renal failure and diabetes mellitus. Nature has endowed each cell with adequate protective mechanisms against any harmful effects of free radicals: SOD, GSH peroxidase, GSH reductase, thioredoxin, thiols and disulfide bonding are the buffering systems in every cell. $\alpha$ Tocopherol vitamin $\mathrm{E}$ (vit $\mathrm{E}$ ) is an essential nutrient that functions as a chain-breaking antioxidant, which prevents the propagation of free radical reactions in all cell membranes in the human body. Ascorbic acid (vit C) is also part of the normal protective mechanism. Other non-enzymatic antioxidants include carotenoids, flavonoids and related polyphenols, $\alpha$-lipoic acid, GSH and so on.

\section{ANTIOXIDANTS IN SCI TREATMENT}

ROS, such as superoxide radical anion, hydroxyl radical and hydrogen peroxide, are produced in metabolic and physiological processes. The oxidative effects of ROS are controlled by exogenous antioxidants such as vit $\mathrm{E}$ and $\mathrm{C}$, and also by endogenous antioxidants such as scavenger enzymes (SOD and GSH peroxidase), bilirubin and uric acid. ${ }^{27}$ When the production of damaging free radicals exceeds the capacity of the body's antioxidant defenses to detoxify them, a condition known as oxidative stress occurs, such that increase in oxidants and decrease in antioxidants cannot be prevented, and the oxidative/antioxidative balance shifts towards the oxidative status. Cellular injury can be caused by oxidative stress, which has been implicated in over a hundred disorders, including SCI. ${ }^{28}$ Interception of free radicals is mainly by radical scavenging, whereas at the secondary level scavenging of peroxyl radicals are affected. The effectors include various antioxidants like vit $\mathrm{C}$ and $\mathrm{E}, \mathrm{GSH}$, other thiol compounds, carotenoids, flavonoids and so on. At the repair and reconstitution level, mainly repair enzymes such as SOD and quinine reductase are involved.

It is believed that compounds that could interrupt the LP process after it has begun would be able to exert a more practical neuroprotective effect (that is, possess longer antioxidant therapeutic window). On the other hand, high-dose oral vit E supplementation for 5 days before SCI acts to attenuate the progressive posttraumatic decrease in white matter spinal cord blood flow together with a significant enhancement in hind-limb motor function compared with non-vit E supplemented animals, this effect has been replicated in a rat compression SCI model. ${ }^{29}$ In contrast, vit E deficiency has been shown to increase post-SCI LP and to attenuate motor functional recovery. ${ }^{30}$ However, despite these effects, the value of vit $\mathrm{E}$ as an acute treatment for SCI is limited by the fact that it requires weeks to achieve a significant increase in parenchymal CNS tissue levels.
On the other hand, long-term, oral high-dose supplementation with vit E may provide effective prophylactic neuroprotection against SCI. Unfortunately, none of the experimental SCI studies have defined the dose response for prophylactic vit E neuroprotection. As a possible guide for human use of vit $\mathrm{E}$ supplementation to limit post-SCI neurological impairment, it has been shown that the dose required to lessen baseline LP product levels in human plasma after 20 weeks of daily supplementation is $800 \mathrm{IU}$ per day. ${ }^{31}$ The author's laboratory tested whether the early administration of high-dose glucocorticoid methylprednisolone (MP) might be able to inhibit posttraumatic spinal cord LP. In an initial set of experiments in cats, it was observed that the administration of an intravenous bolus of MP could indeed inhibit posttraumatic LP in spinal cord tissue, ${ }^{32}$ but that the doses required for this effect were surprisingly high $\left(30 \mathrm{mg} \mathrm{kg}^{-1}\right)$. Further experimental studies, also conducted in cat SCI models, showed that the $30 \mathrm{mg} \mathrm{kg}^{-1}$ dose of MP not only prevented LP, but in parallel, it inhibited posttraumatic spinal cord ischemia and supported aerobic energy metabolism (that is, reduced lactate and improved ATP levels), improved recovery of extracellular calcium (that is, reduced intracellular overload), and attenuated calpain-mediated neurofilament degradation. ${ }^{33}$

Further pharmacological validation of the neuroprotective benefits of inhibition of oxidative damage in the injured spinal cord is indicated by several compounds, which have been reported to attenuate oxidative damage in SCI models. These include methylene blue, mexilitine, thiopental, $\beta$-glucan, cyclosporine A, erythropoietin and $\alpha$-lipoic acid. ${ }^{34}$ Although some of these are probably true chemical antioxidants (for example, methylene blue, edaravone, $\alpha$-lipoic acid), others may work indirectly by induction of endogenous antioxidant defenses (for example, $N$-acetylcysteine), reduction of mitochondrial dysfunction and its associated free-radical leakage (for example, cyclosporine A) or by enhancement of cell membrane repair mechanisms (for example, polyethylene glycol). All these data support the idea that antioxidant therapy may be beneficial in SCI patients. Antioxidant therapies have been proven effective in many pathological processes in which oxidative stress has an important role in $\mathrm{CoQ}_{10}$, vit $\mathrm{E}$, vit $\mathrm{C}$, melatonin, SOD, vit A, GSH, $\mathrm{N}$-acetylcysteine and so on, are some of the antioxidants used in randomized trials of patients with a variety of diseases. However, in the case of SCI, there are still no proven doubleblind and placebo-controlled trials in which the possible mechanisms demonstrate the benefits of these therapies in general and of $\mathrm{CoQ}_{10}$ in particular. Therefore, further studies are necessary in this regard, as well as the design of controlled trials on the therapeutic effect of antioxidants.

\section{CONCLUSION}

Free radical-induced oxidative damage is arguably one of the bestvalidated mechanisms involved in the complex secondary injury cascade, which occurs following acute SCI. Although free radicalmediated damage can occur in all types of biomolecules, LP appears to have a dominant role owing to the high concentration of peroxidation-sensitive polyunsaturated fatty acids and high levels of the LP catalyst iron. Most convincingly, various LP-inhibiting compounds and other antioxidants have been shown to be neuroprotective in acute SCI models. Free radicals have been implicated in the etiology of large number of major diseases including SCI. They can adversely alter many crucial biological molecules leading to the loss of form and function. Such undesirable changes in the body can lead to diseased conditions. Antioxidants can protect against the damage induced by free radicals acting at various levels. Dietary and other components of plants form major sources of antioxidants. The relationship between 
free radicals, antioxidants and functioning of various organs and organ systems is highly complex, and the discovery of 'redox signaling' is a milestone in this crucial relationship. Thus, a clear understanding on the oxidative stress and antioxidant parameters in patients with SCI may provide useful information to augment the understanding of pathophysiology and may also help in routine diagnosis of the patients. However, complete understanding of the biochemical events occurring at a cellular level to influence oxidative damage is required to guide future therapeutic advances. Furthermore, supplementation of the regular treatment with antioxidants, such as vit $\mathrm{C}$ and $\mathrm{E}$ may also be considered in these patients.

\section{CONFLICT OF INTEREST}

The authors declare no conflict of interest.

1 Rolls A, Shechter R, Schwartz M. The bright side of the glial scar in CNS repair. Nat Rev Neurosci 2009; 10: 235-241.

2 Horky LL, Galimi F, Gage FH, Horner PJ. Fate of endogenous stem/progenitor cells following spinal cord injury. J Comp Neurol 2006; 498: 525-538.

3 Bethea JR, Dietrich WD. Targeting the host inflammatory response in traumatic spinal cord injury. Curr Opin Neurol 2002; 15: 355-360.

4 Bruce JH, Norenberg MD, Kraydieh S, Puckett W, Marcillo A, Dietrich D. Schwannosis: role of gliosis and proteoglycan in human spinal cord injury. J Neurotrauma 2000; 17: 781-788.

5 Valko M, Leibfritz D, Moncol J, Cronin MT, Mazur M, Telser J et al. Free radicals and antioxidants in normal physiological functions and human disease. Int J Biochem 2007; 39: 44-84.

6 Esterbauer H, Schaur RJ, Zollner H. Chemistry and biochemistry of 4-hydroxynonenal, malonaldehyde and related aldehydes. Free Radic Biol 1991; 11: 81-128.

7 Dumont RJ, Okonkwo DO, Verma S, Hurlbert RJ, Boulos PT, Ellegala DB et al. Acute spinal cord injury Part I: pathophysiologic mechanisms. Clin Neuropharmacol 2001 24: 254-264.

8 Davies KJ. Oxidative stress: the paradox of aerobic life. Biochem Soc Symp 1995; 61: $1-31$.

9 Fibach E, Rachmilewitz E. The role of oxidative stress in hemolytic anemia. Curr Mol Med 2008; 8: 609-619.

10 Zwart LL, Meerman JH, Commandeur JN, Vermeulen NP. Biomarkers of free radical damage applications in experimental animals and in humans. Free Radic Biol Med 1999; 26: 202-226.

11 Hall ED. Antioxidant therapies for acute spinal cord injury. Neurotherapeutics 2011; 8: 152-167.

12 Varija D, Kumar KP, Reddy KP, Reddy VK. Prolonged constriction of sciatic nerve affecting oxidative stressors \& antioxidant enzymes in rat. Indian J Med Res 2009; 129 587-592.
13 Hosaka N, Kimura S, Yamazaki A, Wang X, Denda H, Ito T et al. Significant correlation between cerebrospinal fluid nitric oxide concentrations and neurologic prognosis in incomplete cervical cord injury. Eur Spine J 2006; 17: 281-286.

14 Estevez AG, Spear N, Manuel SM, Radi R, Henderson CE, Barbeito L et al. Nitric oxide and superoxide contribute to motor neuron apoptosis induced by trophic factor deprivation. J Neurosci 1998; 18: 923-931.

15 Liu D, Liu J, Wen J. Elevation of hydrogen peroxide after spinal cord injury detected by using the Fenton reaction. Free Radic Biol Med 1999; 27: 478-482.

16 Margaritis I, Rousseau AS, Marini JF, Chopard A. Does antioxidant system adaptive response alleviate related oxidative damage with long term bed rest? Clin Biochem 2009; 42: 371-379.

17 Hall ED, Springer JE. Neuroprotection and acute spinal cord injury: a reappraisal. NeuroRx 2004; 1: 80-100

18 Michael-Titus AT. Omega-3 fatty acids and neurological injury. Prostaglandins Leukot Essent Fatty Acids 2007; 77: 295-300.

19 Hall ED, Vaishnav RA, Mustafa AG. Antioxidant therapies for traumatic brain injury. Neurotherapeutics 2010; 7: 51-61.

20 Kigwell BA. Nitric oxide-mediated metabolic regulation during exercise: effect of training in health and cardiovascular disease. FASEB J 2000; 14: 1685-1696.

21 Cepinskas G, Rui T, Kvietys PR. Interaction between reactive oxygen metabolites and nitric oxide in oxidant tolerance. Free Radic Biol Med 2002; 33: 433-440.

22 Sullivan PG, Thompson MB, Scheff SW. Cyclosporin A attenuates acute mitochondrial dysfunction following traumatic brain injury. Exp Neurol 1999; 160: 226-234.

23 Sullivan PG, Krishnamurthy S, Patel SP, Pandya JD, Rabchevsky AG. Temporal characterization of mitochondrial bioenergetics afterspinal cord injury. J Neurotrauma 2007; 24: 991-999.

24 Xiong Y, Rabchevsky AG, Hall ED. Role of peroxynitrite in secondary oxidative damage after spinal cord injury. J Neurochem 2007; 100: 639-649.

25 Vaishnav RA, Singh IN, Miller DM, Hall ED. Lipid peroxidation derived reactive aldehydes directly and differentially impair spinal cord and brain mitochondrial function. J Neurotrauma 2010; 27: 1311-1320.

26 Yorek MA. The role of oxidative stress in diabetic vascular and neural disease. Free Radic Res 2003; 37: 471-480.

27 Erel 0 . A novel automated method to measure total antioxidant response against potent free radical reactions. Clin Biochem 2004; 37: 112-119.

28 Halliwell B. Free radicals, antioxidants and human disease: curiosity, cause or consequence. Lancet 1994; 344: 721-724.

29 Hall ED, Wolf DL. A pharmacological analysis of the pathophysiological mechanisms of posttraumatic spinal cord ischemia. J Neurosurg 1986; 64: 951-961.

30 Taoka Y, Ikata T, Fukuzawa K. Influence of dietary vitamin E deficiency on compression injury of rat spinal cord. J Nutr Sci Vitaminol 1990; 36: 217-226.

31 Roberts LJ 2nd, Oates JA, Linton MF, Fazio S, Meador BP, Gross MD et al. The relationship between dose of vitamin $E$ and suppression of oxidative stress in humans. Free Radic Biol Med 2007; 43: 1388-1393.

32 Hall ED, Braughler JM. Acute effects of intravenous glucocorticoid pretreatment on the in vitro peroxidation of cat spinal cord tissue. Exp Neurol 1981; 73: 321-324.

33 Braughler JM, Hall ED. Effects of multi-dose methylprednisolone sodium succinate administration on injured cat spinal cord neurofilament degradation and energy metabolism. J Neurosurg 1984; 61: 290-295.

34 Toklu HZ, Hakan T, Celik H, Biber N, Erzik C, Ogunc AV et al. Neuroprotective effects of alpha-lipoic acid in experimental spinal cord injury in rats. J Spinal Cord Med 2010; 33 401-409. 